\title{
BMJ Open Rates and predictors of general practitioner (GP) follow-up postdischarge from a tertiary hospital cardiology unit: a retrospective cohort study
}

Luke Y I Huang, ${ }^{1,2}$ Samuel J Fogarty, ${ }^{2}$ Arnold C T Ng, ${ }^{2}$ William Y S Wang (1) ${ }^{1,2}$

To cite: Huang LYI, Fogarty SJ, $\mathrm{Ng} \mathrm{ACT}$, et al. Rates and predictors of general practitioner (GP) follow-up postdischarge from a tertiary hospital cardiology unit: a retrospective cohort study. BMJ Open 2019;9:e031627. doi:10.1136/ bmjopen-2019-031627

- Prepublication history and additional material for this paper are available online. To view these files, please visit the journal online (http://dx.doi. org/10.1136/bmjopen-2019031627).

Received 13 May 2019 Revised 27 September 2019 Accepted 08 0ctober 2019

Q Check for updates

(c) Author(s) (or their employer(s)) 2019. Re-use permitted under CC BY-NC. No commercial re-use. See rights and permissions. Published by BMJ.

${ }^{1}$ Faculty of Medicine, University of Queensland, Brisbane, Queensland, Australia

${ }^{2}$ Department of Cardiology, Princess Alexandra Hospital, Brisbane, Queensland, Australia

Correspondence to Dr William Y S Wang; william.wang@uq.edu.au

\section{ABSTRACT}

Objective Previous studies in cardiac patients noted that early patient follow-up with general practitioners (GPs) after hospital discharge was associated with reduced rates of hospital readmissions. We aimed to identify patient, clinical and hospital factors that may influence GP followup of patients discharged from a tertiary cardiology unit. Design Single centre retrospective cohort study. Setting Australian metropolitan tertiary hospital cardiology unit.

Participants 1079 patients discharged from the hospital cardiology unit within 3 months from May to July 2016. Outcome measures GP follow-up rates (assessed by telephone communication with patients' nominated GP practices), demographic, clinical and hospital factors predicting GP follow-up.

Results We obtained GP follow-up data on 983 out of 1079 (91.1\%) discharges in the study period. Overall, 7 , 14 and 30 -day GP follow rates were $50.3 \%, 66.5 \%$ and $79.1 \%$, respectively. A number of patient, clinical and hospital factors were associated with early GP followup, including pacemaker and defibrillator implantation, older age and having never smoked. Documented recommendation for follow-up in discharge summary was the strongest predictor for 7-day follow-up $(p<0.001)$. Conclusion After discharge from a cardiology admission, half of the patients followed up with their GP within 7 days and most patients followed up within 30 days. Patient and hospital factors were associated with GP follow-up rates. Identification of these factors may facilitate prospective interventions to improve early GP follow-up rates.

\section{INTRODUCTION}

Early follow-up of patients discharged from hospitals by general practitioners (GPs) or other primary care physicians is essential in assuring continuity of care and associated with lower rates of hospital readmissions. ${ }^{1}$ Shared models of care between GPs and hospital specialists have demonstrated improved outcomes in patients with long-term chronic diseases such as heart failure and diabetes. ${ }^{2-4}$ The cooperation between hospital specialists
Strengths and limitations of this study

- This is a large retrospective study involving 1079 patients.

- Identification of discharge planning factors predicting early general practitioner follow-up paves way for future prospective studies.

- Conclusions drawn from a single centre study may not be generalisable to other sites.

- Study conducted over May 2016 to July 2016 did not take into seasonal variations.

and GPs in the community is important to ensure a smooth transition for patients after acute hospital admissions to facilitate successful continued treatment of disease in the community and prevent hospital readmissions. ${ }^{56}$

A recent data-matching study of two Australian states noted that patients who had a GP follow-up within 1 week after hospital discharge for cardiovascular disease had a $5 \%$ lower rate of emergency readmission compared with those who visited a GP after 30 days. ${ }^{1}$ A prospective multicentre study of heart failure patients demonstrated that assessment of patients within 7 days by cardiologists, GPs or heart failure nurses postdischarge significantly reduced the rates of hospital readmissions. ${ }^{7}$

Early GP follow-up helps to ensure that patients receive appropriate care postdischarge. Patients are most vulnerable during the initial days postdischarge due to changes in their therapies. ${ }^{8}$ Early GP follow-up could assist patients in medication adherence, provide ongoing education and support, and address any problems with navigating the healthcare system. ${ }^{9}$

A few studies have provided predictors to early GP follow-up posthospital discharge 
in cardiac patients. After admissions with heart failure, patients whose primary place of residence were in an area with high physician concentration had a higher rate of follow-up within 7 days postdischarge. Patients living in rural/remote areas and patients with lower social economic status were less likely to follow-up within 7 days of discharge, which may reflect geographical and financial barriers to access primary care. Furthermore, heart failure patients with other comorbidities including chronic kidney disease and chronic obstructive pulmonary disease (COPD) were less likely to receive 7-day follow-up. A possible explanation is that patients with more comorbidities may have more difficulty arranging physician visits due to social reasons and they are also more likely to suffer adverse outcomes within that 7 days. ${ }^{10}$

Studies in the USA have also noted that race and gender are associated with difference in GP follow-up rates. Women presenting with heart failure were found to have lower rate of early 7-day follow-up and a subsequent higher risk of 30-day readmission. ${ }^{10}{ }^{11}$ Following a cardiology admission, African-American patients were less likely to have primary care follow-up within 7 days, ${ }^{12} 13$ and more likely to be readmitted within 30 days of discharge.

While demographic and patient clinical contributors to GP follow-up have been identified, the association between hospital activities and GP follow-up is less well studied. In particular, it is unclear if discharge planning efforts made by hospital to recommend GP follow-up is of much effect. To further understand the rates and predictors of GP follow-up, we performed a retrospective analysis of patients postdischarge from the cardiology unit of a tertiary hospital in Australia. We focused on 7 and 30-day windows to be consistent with the current literature.

\section{METHODS}

\section{Patient cohort}

To assess rates and predictors for GP follow-up of patients postdischarge from a tertiary cardiology unit, we retrospectively reviewed medical records of all patients discharged the Department of Cardiology at Princess Alexandra Hospital (PAH), Brisbane, Australia, for 3-month period from 1 May 2016 to 31 July 2016. PAH is a metropolitan tertiary hospital treating patients from within the local health services area and also from rural and remote centres. Exclusion criteria included patients who were involved in other programme or trials that would affect GP follow-up after discharge, patients who died in hospital, patients who were discharged back into correctional facility and patients who had no documented community GP.

\section{Study design}

Medical records were audited to identify demographic details, reasons for admission, hospital procedures performed, background medical history and discharge planning factors. Discharge planning factors included documentation in medical records for GP follow-up, documentation in medical records for specialist follow-up, documentation in discharge summaries recommending GP follow-up and documentation in discharge summaries recommending specialist follow-up. GP follow-up information was obtained by telephoning individual GP practices at least 2 months after date of discharge based on GP practice details in hospital records. GP follow-up rates within 7 and 30 days of discharge were determined. Association between patient, hospital and discharge planning factors and GP follow-up rates were determined.

\section{Statistical analysis}

Continuous variables were presented as the mean \pm SD. Categorical variables were presented as frequencies or percentages. Continuous variables were tested for normality. Differences between two groups for normally distributed variables were tested using Student's t-test. The Mann-Whitney U tests were used to analyse continuous variables. $\mathrm{X}^{2}$ tests were used to analyse categorical variables. A $p<0.05$ was considered statistically significant. Variables with $\mathrm{p}<0.1$ from univariable analysis were selected for the multivariable analysis. Multivariate linear regression analyses, with a backward-elimination approach, were performed to identify the variables with the strongest association with GP follow-up parameters. All statistical calculations were performed using IBM SPSS Statistics for Windows, V.21.0.

\section{Patient and public involvement}

Patients and public were not involved in the design and planning of the study.

\section{RESULTS}

A total of 1079 patients who met the inclusion criteria were discharged from PAH cardiology between May 2016 and July 2016. GP follow-up data were obtained for 983 of them $(91.1 \%)$.

Patients' background characteristics, demographics, medical background are summarised in table 1. There were more male patients discharged during this period $(68.4 \%)$. The mean age of patients was $63.7 \pm 14.2$. Only $34.5 \%$ of patients were employed in full-time work at the time of hospital admission and only $18.4 \%$ of patients had private health insurance.

Patients' causes of presentation and the procedures performed during inpatient stay are summarised in table 2. Common diagnoses included $20.7 \%$ of patients with either ST or non-ST elevation myocardial infarction (STEMI or NSTEMI) and $19.9 \%$ of patients had cardiac arrhythmia. Heart failure presentation only formed $9.6 \%$ of total presentations. The most common diagnosis was non-cardiac chest pain at $24.4 \%$.

\section{GP follow-up rates}

A total of 494 cardiology patients followed up with their GP within 7 days of discharge (50.3\%) and $778(79.1 \%)$ followed up within 30 days. Over half of discharge summaries $(58.8 \%)$ were completed within 7 days and over two-thirds $(67.8 \%)$ were completed within 30 days. 
Table 1 Patientdemographics and medical history

\begin{tabular}{|c|c|}
\hline Characteristics & Patients $(n=983)$ \\
\hline Male, n (\%) & $672(68.4)$ \\
\hline Age (mean $\pm S D)$ & $63.7 \pm 14.2$ \\
\hline Distance to hospital (km, median (range)) & $23(0-4390)$ \\
\hline Married, n (\%) & $588(57.9)$ \\
\hline Current employment, n (\%) & $338(34.5)$ \\
\hline \multicolumn{2}{|l|}{ Smoking status, n (\%) } \\
\hline Non-smoker & $381(41.9)$ \\
\hline Ex-smoker & $347(38.1)$ \\
\hline Current smoker & $182(20.0)$ \\
\hline English speaking, n (\%) & $879(89.5)$ \\
\hline Private health insurance, $\mathrm{n}(\%)$ & $181(18.4)$ \\
\hline \multicolumn{2}{|l|}{ Medical history } \\
\hline Ischaemic heart disease, n (\%) & $483(49.4)$ \\
\hline Arrhythmia, n (\%) & $341(34.9)$ \\
\hline Congestive cardiac failure, $\mathrm{n}(\%)$ & $165(16.9)$ \\
\hline Hypertension, n (\%) & $564(57.7)$ \\
\hline Hypercholesterolaemia, n (\%) & $417(42.6)$ \\
\hline Diabetes, n (\%) & $286(29.2)$ \\
\hline
\end{tabular}

No monthly differences were noted between May, June and July 2016.

\section{Predictors of GP follow up}

Independent predictors of GP follow-up in 7 days and 30 days from multivariable regression analysis are shown in tables 3 and 4, respectively. Univariable analysis results are shown in online supplementary appendix. A mix of

\begin{tabular}{lc}
\hline $\begin{array}{l}\text { Table } 2 \\
\text { during admission }\end{array}$ & Neason (\%) \\
\hline Clinical factors & \\
\hline Diagnosis & $52(5.3)$ \\
\hline ST and non-ST elevation myocardial infarct & $203(20.7)$ \\
\hline Angina/unstable angina & $94(9.6)$ \\
\hline Heart failure & $196(19.9)$ \\
\hline Arrhythmia & $39(4.0)$ \\
\hline Other cardiac cause & $240(24.4)$ \\
\hline Non-cardiac chest pain & $8(0.8)$ \\
\hline Other non-cardiac cause & $161(16.4)$ \\
\hline Type of admission & $213(21.7)$ \\
\hline Interhospital transfer & \\
\hline Elective admission & $303(30.8)$ \\
\hline Procedures performed & $190(19.3)$ \\
\hline Coronary angiogram & $97(9.9)$ \\
\hline Coronary stents & \\
\hline $\begin{array}{l}\text { Pacemaker/implantable defibrillator } \\
\text { insertion }\end{array}$ & \\
\hline
\end{tabular}

demographic, clinical and discharge planning factors independently predicted GP follow-up.

The hospital discharge planning factor, which independently predicted GP follow-up within 7 days, was discharge summary recommendation of GP follow-up. Other positive predictors for 7-day follow-up were patients who had cardiac pacemaker and defibrillator implanted, patients who presented with heart failure and patients who were transferred from other hospital. Smoking status was a negative predictor of GP follow-up (table 3 ).

The hospital discharge planning factors predicting GP follow-up at 30 days were medication changes during admission and medical records noting recommendation for GP follow-up, but not documentation in discharge summaries (table 4). Other positive and negative predictors of GP follow-up within 30 days are presented in table 4 .

Patients who have ever smoked consisted of current smokers and ex-smokers. We first used ever smoked status for our multivariate logistic regression analyses. When analyses were repeated using current smokers instead of ever smoked, the results were similar.

\section{DISCUSSION}

\section{GP follow-up rates}

Our GP follow-up rate within 7 days of $50.3 \%$ is broadly consistent with that of other Australian states, ${ }^{1}$ but higher compare to the large multihospital study done in the USA for heart failure patients by Kociol et al, which noted $37.9 \%$ of patients have early GP follow-up within 7 days. ${ }^{10}$ Our cohort consisted of all cardiology admissions instead of heart failure alone, although we did note that patients with heart failure presentations were more likely to be followed up with their GPs within 7 days compared with other patients (table 3). Different healthcare structures between USA and Australia may contribute to these differences. In the USA, patients are generally followed up by the treating physicians postdischarge whereas in Australia most patients are followed up in the short term by GPs in the community with hospital specialist follow-up at a later date. $^{14}$

We noted GP follow-up rates of $79.1 \%$ of within 30 days of discharge. At present most available literature examines effects of early discharge follow-up (within 7 days) on 30-day readmission back to hospital.

\section{Patient demographic factors}

One strong negative predictor for both 7 and 30days follow-up identified in this study was patients' smoking status (tables 3 and 4). Smokers are generally less compliant to medical therapy and less likely to be healthconscious compared with non-smokers. ${ }^{15}$ However, smoking is also associated with socioeconomic status and race, information that is not routinely collected in our hospital records. It is possible that these and other unmeasured confounders can potentially affect GP follow-up rate and smoking status. 
Table 3 Independent predictors of GP follow-up within 7 days of discharge

\begin{tabular}{|c|c|c|c|}
\hline Predictor & OR $(95 \% \mathrm{Cl})$ & $P$ value & Association \\
\hline Pacemaker/implantable defibrillator insertion & 4.52 (2.25 to 8.670$)$ & $<0.001$ & Positive \\
\hline Interhospital transfer & $1.97(1.32$ to 2.94$)$ & 0.001 & Positive \\
\hline Presentation with heart failure & 1.60 (0.98 to 2.61$)$ & 0.058 & Positive \\
\hline Ever smoked & 0.48 (0.36 to 0.64$)$ & 0.001 & Negative \\
\hline
\end{tabular}

GP, general practitioner.

Increased age was a positive predictor of GP follow-up within 30 days (table 4). Older patients are more likely to have more complex medical comorbidities. ${ }^{6}$ GP follow-up postdischarge can serve to optimise patients' medical comorbidities and assist them in transition from hospital to home. On the contrary, a previous study by Kociol et $a l^{10}$ noted that patients with high-risk comorbidities such as kidney disease and COPD have a lower follow-up rate.

In this study, gender was not identified to be a positive nor negative predictor of early GP follow-up whereas in a previous study female gender was a negative predictor for early GP follow-up postdischarge for heart failure and a higher risk of 30-day readmission. ${ }^{10}$ The difference may be due to the variety of conditions in our cohort, rather than the isolated heart failure group.

\section{Patient clinical factors}

The strongest positive predictor of early GP follow-up in this study was having permanent pacemaker or implantable cardioverter-defibrillator implanted during admission. Patients who had devices implanted routinely follow-up early with their GP for removal of dressings and assessment of the wound. Most of these patients were admitted electively for the procedure and discharged the next day. Hence majority of these patients had documented discharge summary recommendations for GP to monitor the patient for any postoperative complications. Early GP follow-up for patients post implantable cardioverter/pacemaker implantation could also prompt review of any issues related to the device itself. ${ }^{16}$
Patients who were transferred from other hospitals to our tertiary centre for management of their cardiac issues were more likely to follow-up with their GP within 7 days of discharge $(p<0.001)$. These patients were generally sicker with higher proportion of STEMI and NSTEMI requiring coronary intervention, or had high-risk arrhythmias or conduction disease. They were more likely to see their GP for medical optimisation early on discharge possibly because of the severity of their presenting illness, a result consistent with previous findings. ${ }^{7}$

Other positive predictors of GP follow-up included patients with medical history of ischaemic heart disease ( $\mathrm{p}=0.042)$ and patients who presented with STEMI or NSTEMI $(p<0.001)$. Patients with medical history of ischaemic heart disease often have more medical comorbidities compared with those without history of ischaemic heart disease.$^{17}$ It can be postulated that these patients are more likely to follow up with their local GP early postdischarge for optimisation of their more complex comorbidities. They are also more likely to be on more medications than patients without history of ischaemic heart disease. ${ }^{18}$ GP follow-up postdischarge can help patients understand and adhere to any changes to these medications made during inpatient hospital stay.

Patients who presented with STEMI/NSTEMI often required interventional procedures. They are routinely prescribed evidence-based medications, and may require lifestyle changes such as smoking cessation and dietary changes. This could explain why we found these patients

\begin{tabular}{|c|c|c|c|}
\hline Predictor & OR $(95 \% \mathrm{Cl})$ & $P$ value & Association \\
\hline Pacemaker/implantable defibrillator insertion & $4.72(1.75$ to 14.20$)$ & 0.003 & Positive \\
\hline Any medication change during admission & 1.99 (1.31 to 2.91$)$ & $<0.001$ & Positive \\
\hline History of ischaemic heart disease & $1.48(1.01$ to 2.16$)$ & 0.042 & Positive \\
\hline Age & $1.02^{*}(1.00$ to 1.03$)$ & 0.019 & Positive \\
\hline Ever smoked & $0.68(0.46$ to 0.95$)$ & 0.027 & Negative \\
\hline
\end{tabular}

${ }^{\star} \operatorname{Exp}(B)$ for continuous variable.

GP, general practitioner; STEMI/NSTEMI, ST or non-ST elevation myocardial infarction. 
were more likely to see their GP within 30 days of discharge.

In this study, patients heart failure trended towards early follow-up with their GPs within 7 days of discharge. Patients who were admitted with heart failure have been extensively studied previously. Within Medicare data in USA, greater than $20 \%$ of patients hospitalised for heart failure were readmitted within 30 days of discharge, generally with worsening heart failure. ${ }^{1920}$ These patients often need GP input early after discharge to assist with titration of medications and assessment of fluid status. Furthermore, patients who were admitted with heart failure as a group tend to have more medical comorbidities. ${ }^{10}$ Early GP follow-up for heart failure patients is associated with reduced rates of 30 day readmission in the USA. ${ }^{10} 2122$ It has also been shown in prospective Australian multicentre study on patients with heart failure that nurse-led disease management plan together with medical review by GP within 7 days is associated with reduced rate of readmission at 30 and 90 days. $^{7}$

In our study, patients who had medication changes were more likely to follow up with their GP within 30 days of discharge. Our hospital generally supplies only 1-month worth of medications and hence patients have to see their GP for ongoing medication prescriptions. Patients with medication changes also needed to be monitoring for progress and potential side effects.

\section{Discharge planning factors}

We noted that recommending early GP follow-up in discharge summaries was independently associated with higher rates of GP follow-up within 7 days. This association may be explained by the possibility that discharge summaries serve as reminders for both patients and GPs to arrange follow-up medical visits as our hospital routinely sends copies of discharge summary to both patients and their nominated GPs. An alternative explanation would be that the association is due to patients' underlying diseases or treatment plans. A prospective interventional study could potentially determine whether the discharge summaries directly influence rates of early GP follow-up or not.

Discharge summaries are integral to handover of clinical care from hospital specialists to GPs. ${ }^{23}$ Availability of high-quality discharge summaries at discharge has been shown to be associated with a lower risk of readmission back to hospital. ${ }^{24}$ The current rate of completion and availability of pertinent information on discharge summaries in Australian healthcare setting has significant potential for improvement. A Western Australian study showed that only $37 \%$ of discharge summaries contained plan for further outpatient management, while a retrospective audit in New South Wales, Australia, showed that only $27.1 \%$ of discharge summaries were received by the GPs. ${ }^{25} 26$

Documentation in medical record reflects clinicianpatient communications of diagnosis, progress and future plans. In this study, we noted that documentation in hospital medical record for patients to follow up with their GP on discharge was associated with a positive trend with patients visiting their GPs within 30 days of discharge but does not affect follow-up within 7 days. In contrast, discharge summaries recommendations were strongly associated with higher rates of 7-day GP follow-up. This observed difference may hint to the importance of written documentations postdischarge as patients may be overloaded with information during hospital admissions.

\section{Study limitation}

This study is a retrospective single centre study. GP follow-up data were obtained through telephone communication with patients' nominated GP practices. It is possible that patients could have seen other GPs after discharge. Being a retrospective study, it is not possible to determine if the association between early GP follow-up and discharge summaries is due to discharge planning or underlying levels of patients' sickness. A future prospective study is required to resolve this issue.

\section{CONCLUSIONS}

We have identified a number of patient, disease and hospital discharge planning factors associated with GP follow rates. The finding that recommendation for early GP follow in discharge summaries is associated with higher rates of actual early 7-day GP follow-up paves the way for potential prospective studies aimed at improving rates of early GP follow-up and reduce hospital readmissions.

Contributors LH and WW contributed to the study's conception and design. LH acquired the data. LH, SF, AN and WW contributed to the analysis and interpretation of the data. LH and WW drafted the manuscript. LH, SF, AN and WW contributed to the critical revision and approved the manuscript.

Funding The authors have not declared a specific grant for this research from any funding agency in the public, commercial or not-for-profit sectors.

Competing interests None declared.

Patient consent for publication Not required.

Ethics approval Ethics approval was obtained from Metro South Hospital and Health Service Human Research Ethics Committee with reference number of HREC/18/QPAH/228.

Provenance and peer review Not commissioned; externally peer reviewed.

Data availability statement Data are available on reasonable request, but data sharing would be subjected to additional ethics approval.

Open access This is an open access article distributed in accordance with the Creative Commons Attribution Non Commercial (CC BY-NC 4.0) license, which permits others to distribute, remix, adapt, build upon this work non-commercially, and license their derivative works on different terms, provided the original work is properly cited, appropriate credit is given, any changes made indicated, and the use is non-commercial. See: http://creativecommons.org/licenses/by-nc/4.0/.

ORCID iD

William Y S Wang http://orcid.org/0000-0002-5369-5446

\section{REFERENCES}

1 AlHW. Transition between hospital and community care for patients with coronary heart disease: New South Wales and Victoria 20122015. Canberra: AlHW, 2018.

2 Griffin S. Diabetes care in general practice: meta-analysis of randomised control trials. BMJ 1998;317:390-6. 
3 Ahmed A, Allman RM, Kiefe Cl, et al. Association of consultation between generalists and cardiologists with quality and outcomes of heart failure care. Am Heart $J$ 2003;145:1086-93.

4 Ezekowitz JA, van Walraven C, McAlister FA, et al. Impact of specialist follow-up in outpatients with congestive heart failure. CMAJ 2005;172:189-94.

5 HANSEN FR, SPEDTSBERG K, Schroll M. Geriatric follow-up by home visits after discharge from hospital: a randomized controlled trial. Age Ageing 1992;21:445-50.

6 Overland J, Yue DK, Mira M. Continuity of care in diabetes: to whom does it matter? Diabetes Res Clin Pract 2001;52:55-61.

7 Huynh Q, Negishi K, De Pasquale C, et al. Effects of post-discharge management on rates of early re-admission and death after hospitalisation for heart failure. Med J Aust 2018;208:485-91.

8 Leendertse AJ, Egberts ACG, Stoker LJ, et al. Frequency of and risk factors for preventable medication-related hospital admissions in the Netherlands. Arch Intern Med 2008;168:1890-6.

9 Balaban RB, Williams MV. Improving care transitions: hospitalists partnering with primary care. J Hosp Med 2010;5:375-7.

10 Kociol RD, Greiner MA, Fonarow GC, et al. Associations of patient demographic characteristics and regional physician density with early physician follow-up among medicare beneficiaries hospitalized with heart failure. Am J Cardiol 2011;108:985-91.

11 Howie-Esquivel J, Dracup K. Effect of gender, ethnicity, pulmonary disease, and symptom stability on rehospitalization in patients with heart failure. Am J Cardiol 2007;100:1139-44.

12 Sonel AF, Good CB, Mulgund J, et al. Racial variations in treatment and outcomes of black and white patients with high-risk non-STelevation acute coronary syndromes: insights from crusade (can rapid risk stratification of unstable angina patients suppress adverse outcomes with early implementation of the ACC/AHA guidelines?). Circulation 2005;111:1225-32.

13 Skinner J, Chandra A, Staiger D, et al. Mortality after acute myocardial infarction in hospitals that disproportionately treat black patients. Circulation 2005;112:2634-41.

14 De Lew N, Greenberg G, Kinchen K. A layman's guide to the U.S. health care system. Health Care Financ Rev 1992;14:151-69.
15 Castro FG, Newcomb MD, McCreary C, et al. Cigarette smokers do more than just smoke cigarettes. Health Psychol 1989;8:107-29.

16 Korte T, Jung W, Ostermann G, et al. Hospital readmission after transvenous cardioverter/defibrillator implantation; a single centre study. Eur Heart J 2000;21:1186-91.

17 Haffner SM, Lehto S, Rönnemaa T, et al. Mortality from coronary heart disease in subjects with type 2 diabetes and in nondiabetic subjects with and without prior myocardial infarction. N Engl J Med 1998;339:229-34.

18 DeLia D, Tong J, Gaboda D, et al. Post-Discharge follow-up visits and hospital utilization by Medicare patients, 2007-2010. Medicare Medicaid Res Rev 2014;4.10.5600/mmrr.004.02.a01

19 Rohr R. Rehospitalizations among patients in the Medicare fee-forservice program. N Engl J Med 2009;361:311-2. Author reply 2.

20 Dharmarajan K, Hsieh AF, Lin Z, et al. Diagnoses and timing of 30-day readmissions after hospitalization for heart failure, acute myocardial infarction, or pneumonia. JAMA 2013;309:355-63.

21 Lee KK, Yang J, Hernandez AF, et al. Post-discharge follow-up characteristics associated with 30-day readmission after heart failure hospitalization. Med Care 2016;54:365-72.

22 Tung Y-C, Chang G-M, Chang H-Y, et al. Relationship between early physician follow-up and 30-day readmission after acute myocardial infarction and heart failure. PLoS One 2017;12:e0170061.

23 Reid DB, Parsons SR, Gill SD, et al. Discharge communication from inpatient care: an audit of written medical discharge summary procedure against the new national health service standard for clinical handover. Aust Health Rev 2015;39:197-201.

24 van Walraven C, Seth R, Austin PC, et al. Effect of discharge summary availability during post-discharge visits on hospital readmission. J Gen Intern Med 2002;17:186-92.

25 Wilson S, Ruscoe W, Chapman M, et al. General practitioner-hospital communications: a review of discharge summaries. J Qual Clin Pract 2001;21:104-8.

26 Tan B, Mulo B, Skinner M. Transition from hospital to primary care: an audit of discharge summary - medication changes and follow-up expectations. Intern Med J 2014;44:1124-7. 